\title{
Photonic crystals with controlled disorder
}

\author{
P. D. García, ${ }^{1,}{ }^{*}$ R. Sapienza,,${ }^{1, \dagger}$ C. Toninelli, ${ }^{2}$ C. López, ${ }^{1}$ and D. S. Wiersma ${ }^{2}$ \\ ${ }^{1}$ Instituto de Ciencia de Materiales de Madrid (CSIC) and Unidad Asociada CSIC-UVigo, Cantoblanco E-28049, Madrid, Spain \\ ${ }^{2}$ European Laboratory for Nonlinear Spectroscopy \& CNR-INO, 50019 Sesto Fiorentino, Florence, Italy
}

(Received 5 November 2010; published 10 August 2011)

\begin{abstract}
Photonic crystals are extremely sensitive to structural disorder even to the point of completely losing their functionalities. While, on one side, this can be detrimental for applications in traditional optical devices, on the other side, it gives also rise to very interesting new physics and maybe even new applications. We propose a route to introduce disorder in photonic crystals in a controlled way by creating a certain percentage of vacancies in the lattice. We show how the method works and what type of materials can be obtained this way. Also, we use this system to probe the role of disorder on the resulting transport properties from various points of view, including measurements of the transport and scattering mean free path and the diffusion constant.
\end{abstract}

DOI: 10.1103/PhysRevA.84.023813 PACS number(s): 42.25.Dd, 42.25.Bs, 42.25.Fx, 06.30.Gv

\section{INTRODUCTION}

Numerous applications of photonic crystals [1,2] have been proposed based on their ability to control ballistic light transport. In practice, most photonic crystals contain a certain amount of intrinsic disorder which gives rise to multiple light scattering and light diffusion [3]. An extraordinary progress has been made in the fabrication of nanophotonic structures with many novel optical properties [4]. Using engineered disorder by introducing defects of controlled amount, position, shape, and other morphological characteristics can, however, lead to interesting new functionalities [5,6]. The extreme case of disorder is that of a photonic glass in which the building blocks are perfect spheres which are distributed randomly [7]. In such systems, light undergoes multiple resonant Mie scattering due to the equal shape and size of its scatterers [8].

While light propagation in photonic crystals is described by Bloch modes, transport in photonic glasses is dominated by random multiple scattering. Random systems also exhibit interference effects of which maybe the most dramatic is that of Anderson localization of light waves, in which interference brings light transport to a complete halt [9]. The combination of a photonic crystal and a certain amount of random multiple scattering is believed to be the key to observe optical Anderson localization [2]: the presence of a photonic band structure can lead to a strongly reduced diffusion constant compared to an equivalent random system without the underlying periodic backbone [10]. While transverse localization has already been observed in photonic lattices [11], the interplay between order and disorder is mostly unknown, even at low refractive index, far away from the predicted Anderson localization transition.

In this paper we show a method to obtain structures in between a photonic crystal and a photonic glass by adding a small amount of controlled defects and we characterize the optical properties of the resulting topology. The novelty here is the possibility to effectively tune the disorder parameter

\footnotetext{
*Present address: DTU Fotonik, Department of Photonics Engineering, Technical University of Denmark, rsteds Plads 343, DK2800 Kgs. Lyngby, Denmark.

${ }^{\dagger}$ Present address: ICFO-Institut de Ciencies Fotoniques, Mediterranean Technology Park, E-08860 Castelldefels, Barcelona, Spain.
}

in 3D photonic crystal which allow us to probe the optical properties of structures in between order and disorder. By doing so, we are able to study how the controlled introduction of disorder progressively destroys the periodic backbone thus engineering the number of scattering events light suffers across an actual sample of any given thickness and tuning the scattering parameters as the mean free path and the diffusion constant. The paper is organized as follows: In Sec. II we will show how to introduce extrinsic disorder in photonic crystals as vacancies in the lattice and how to analyze the topological disorder of such structures. In Sec. III we will show measurements of the static transport properties, in particular the scattering mean free path. In Sec. IV we will report on measurements of the diffusion constant in photonic crystals as a function of the density of vacancies. In Sec. V we will show total white light transmission measurements from photonic glasses and photonic crystals with a high amount of vacancies.

\section{VACANCY-DOPED PHOTONIC CRYSTALS}

\section{A. Sample preparation}

An alloy photonic crystal [12-14] is grown with a binary colloid that consists of spheres of two types. Compared to the crystals which are composed by single-specie spheres, binary crystals exhibit a rather rich phase behavior that depends on the volume fractions of the constituents, in particular on their diameter ratio. If the constituents are chemically different but of the same diameter, it is possible to also obtain a regular lattice of which, after crystal growth, one of the constituents can be chemically removed. The one constituent that is removed acts in that sense as a dopant, since it introduces vacancies in the lattice. Figures 1(a), 1(b), 1(d), and 1(e) schematize the process. Binary colloidal suspensions of polymethyl-methacrylate (PMMA) and polystyrene (PS) spheres were ordered by vertical deposition [15]. This method allows us to grow large homogenous 3D alloys with fcc crystalline structure and appreciable thickness. While the growth process fixes the crystalline structure, with this method we introduce disorder without altering the particular crystalline structure, by adding a controlled amount of vacancies in the lattice. The total colloidal concentration in the liquid is typically set fixed at $0.15 \mathrm{wt} \%$, and the density of dopants 
(a)

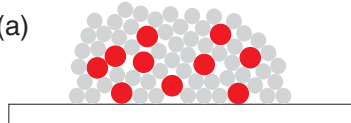

(b)
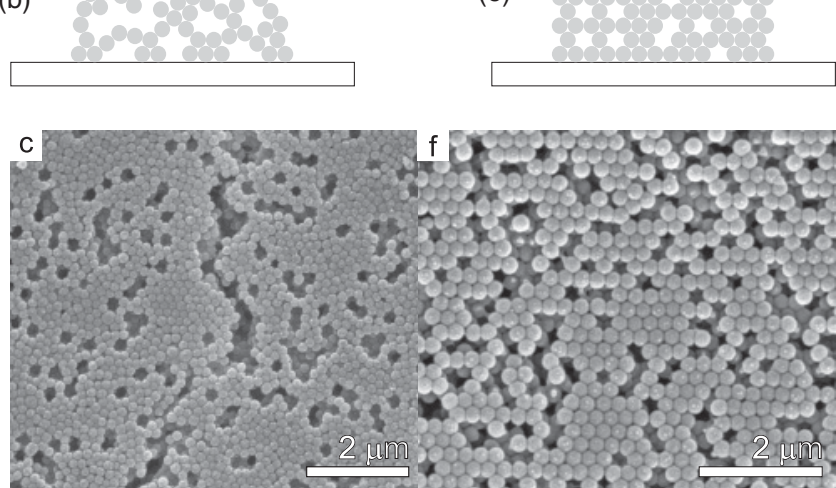

FIG. 1. (Color online) (a) The diagram schematizes the film growth by vertical deposition when polystyrene spheres (PS, red) and polymethyl-methacrylate spheres (PMMA, gray) are mixed together in the initial colloidal suspension with different diameters. The structure that forms depends on the diameter ratio of the spheres and is generally random. By etching with cyclohexane the PS spheres can be removed (b). In (c) a SEM image is shown of such structure. (d), (e), and (f) schematize the same process when both PS and PMMA spheres have the same diameter. In that case a regular lattice is formed with a controlled number of vacancies. In (f) a sample is shown that was grown by using PS and PMMA spheres with diameters $237 \mathrm{~nm}$.

(PS spheres, in this case) is tuned by changing the partial PS concentration in the initial colloidal suspension. For example, if a $10 \%$ final vacancy density is needed, a partial PS spheres concentration of $0.015 \mathrm{wt} \%$ and PMMA concentration of $0.135 \mathrm{wt} \%$ will be mixed to obtain a total $0.15 \mathrm{wt} \%$ colloidal suspension. Once the alloy colloidal crystal is grown, the PS spheres are removed by selective chemical etching by immersing the samples in $99 \%$ pure cyclohexane for at least $4 \mathrm{~h}$. This very easy procedure completely removes the PS spheres, leaving the PMMA spheres undisturbed. Scanning electron microscopy (SEM) image Fig. 1(f) shows a colloidal crystal realized with $30 \%$ of PS spheres and 70\% of PMMA spheres after PS etching. In that case, both PMMA and PS spheres had the same diameter $d=237 \mathrm{~nm}$, with an error included in the polydispersity of each of the spheres $(<2 \%$ of the sphere diameter).

The selective etching of PS spheres can be conveniently monitored optically during the etching process. At low energies, far from the first stopband, the sample can be considered as a homogenous thin layer with effective refractive index $n_{\text {eff }}$. This $n_{\text {eff }}$ can be measured by recording the spectral separation of the Fabry-Perot fringes in the transmittance or reflectance spectra, which depend on the optical thickness of the sample and thereby both on its physical thickness and refractive index. A Fourier transform infrared spectrometer (Bruker IFS-66/S) was used to record the optical reflectance of the samples at normal incidence in a wide spectral range. A microscope attached to the spectrometer with a $\times 4$ objective featuring a 0.1 numerical aperture, that is, $\pm 6^{\circ}$ angular aperture, was used to focus and collect the light. Local reflectance maxima of the
Fabry-Perot fringes will appear at (for an opal on a substrate with a refractive index higher than that of the opal [16])

$$
m \lambda_{m}=2 L n_{\mathrm{eff}} \Longrightarrow \frac{1}{\lambda_{m}}=\frac{m}{2 L n_{\mathrm{eff}}},
$$

where $m$ is the resonance order, $L$ is the sample thickness, and $\lambda_{m}$ is the wavelength of the $m$ resonance. A linear relation is obtained by plotting the inverse of $\lambda_{m}$ as a function of $m$, which slope yields the inverse of the sample thickness and $n_{\text {eff }}$. The initial $n_{\text {eff }}$ of the alloy crystal, composed by PS and PMMA spheres [Fig. 1(d)], can be calculated from the partial concentration of each type of spheres, their refractive index $n_{\mathrm{PS}}=1.59$ and $n_{\mathrm{PMMA}}=1.4$, and the total filling fraction of spheres in the crystal $f=0.74$. This gives us a precise estimation of the sample thickness, which remains constant during the etching process. The evolution of the etching can then be followed by monitoring the value of $n_{\text {eff }}$ with time, using Eq. (1) with this initial value of $L$. Figure 2 shows the effective refractive index for a thin film initially composed of $60 \%$ of PMMA spheres and $40 \%$ of PS spheres, both of diameter $d=237 \mathrm{~nm}$. Figure 2 reveals that the etching is complete after a few minutes, when $n_{\text {eff }}$ reaches a constant value. The inset of Fig. 2 shows the reflectance spectrum from the composite before (black solid curve) and after (red dashed curve) complete PS removal. Apart from the disappearance of the reflectance peak related to the first stopband in the $\Gamma L$ direction (which will be the object of attention in the following section), a blue shift in the Fabry-Perot fringes clearly shows the significant change in $n_{\text {eff }}$. The final vacancy-doped photonic crystals [Fig. 1(f)] have typically the same dimensions as a thin photonic crystal

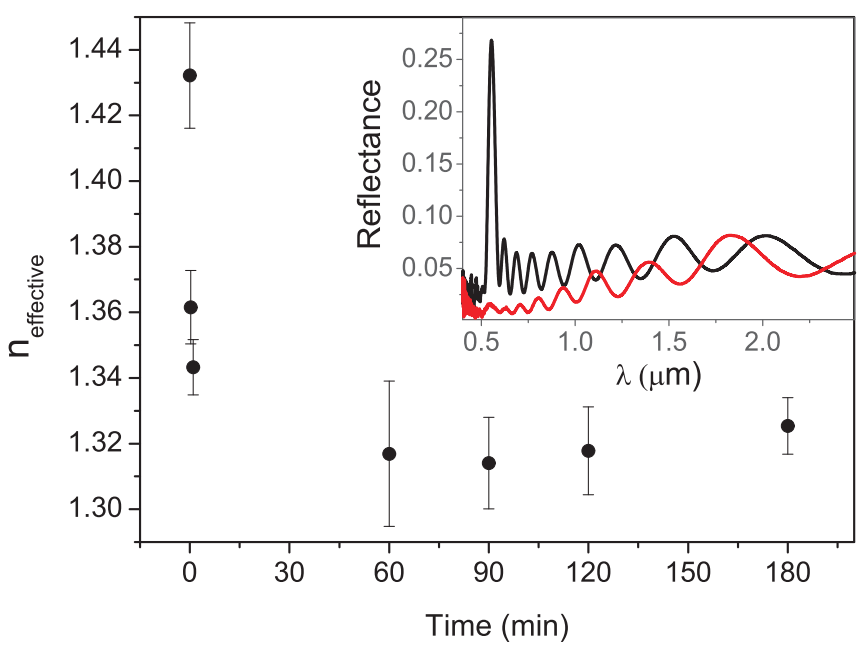

FIG. 2. (Color online) Effective refractive index $\left(n_{\text {eff }}\right)$ of the system as a function of PS etching time The concentration of PS and PMMA spheres are known and the thickness of the sample $L$ can be then calculated from the Fabry-Perot fringes in the reflectance spectrum (black solid curve in the inset of the figure) By dissolving the PS spheres, $n_{\text {eff }}$ reduces and its variation is accounted for by the blueshift in the Fabry-Perot fringes The evolution of $n_{\text {eff }}$ can be estimated with the help of $L$ and the Fabry-Perot fringes of the etched sample (red dashed curve in the inset of the figure) The PS removal has a characteristic time of about few minutes for which $n_{\text {eff }}$ reaches a constant value. 
film, that is, they have a thickness of several microns and can be several centimeters wide. The vacancies are distributed homogeneously throughout the structure.

\section{B. Optical characterization}

As a first characterization of our samples, we have recorded standard angular dependent reflection and transmission spectra. In particular, we recorded the specular reflectance $R$ and transmittance $T$ of light with wavelength from $400 \mathrm{~nm}$ to $3 \mu \mathrm{m}$ in the direction perpendicular to the sample surface $(\Gamma L)$ and in the low-energy range $(a / \lambda<1$, where $a$ is the lattice parameter). This measurement directly gives the amount of light lost from this particular direction which, due to elastic scattering, propagates in other directions different than the incident one. By increasing the amount of vacancies in the crystal lattice, the amount of light scattering increases as we will discuss later.

The analysis carried out in the present section will tackle the measurement of the amount of remaining order in the bulk of the structure. The magnitude $R+T$ can be used to estimate the amount of remaining order $[17,18]$ if absorption and higher order Bragg scattering can be neglected. In that case, the diffuse light intensity $D$ is simply $D=1-R-T$, which accounts for the losses due to elastic light scattering from the lattice vacancies acting as the scatterers [19]. Note that one expects the diffuse intensity to also be nonzero for samples without intentionally introduced defects (our samples with zero percent vacancies), due to residual polydispersity and various sample imperfections that are common for even the most accurately prepared photonic crystal opals [3].

Figures 3(a) and 3(b) show $R$ and $T$ measurements from samples doped with $0 \%$ and $40 \%$ vacancies, respectively. The disappearance of the reflectance (transmittance) peak (dip) related to the pseudogap in the $\Gamma L$ direction is the first and most evident effect of increased disorder. Figure 3(c) plots $D$, proportional to the light loses, which presents the usual features for a finite opal [17]: a monotonic increase for frequencies outside the gap, attributed to Rayleigh-Gans type of scattering, the presence of a dip at $a / \lambda \sim 0.6$ for polymeric spheres which accounts for a reduction of scattering losses inside the gap, and two peaks at the band edges which represents an increase of scattering at these spectral positions. Here we are measuring light which is lost from the initial direction and diffuses through the crystal. Therefore, the asymmetry between the high- and the low-energy band edges can be explained by examining the photonic band structure in other crystallographic directions close to the incident one. For light incident in the $\Gamma L$ direction, scattered photons in the low-energy band edge find allowed states in adjacent directions when a small momentum is acquired. At the high-energy band edge, the additional momentum needed for a scattered photon to couple to a Bloch mode in other directions is larger and the process for this energy becomes less probable than the former. This is the reason which, qualitatively, explains this asymmetry. For a high amount of vacancies, Fig. 3(d) reveals the disappearance of any hint of gap or band-edges features and shows the increase of scattering losses for all frequencies. The higher the relative concentration of PS, the more abrupt the transition is when increasing disorder, constructive and
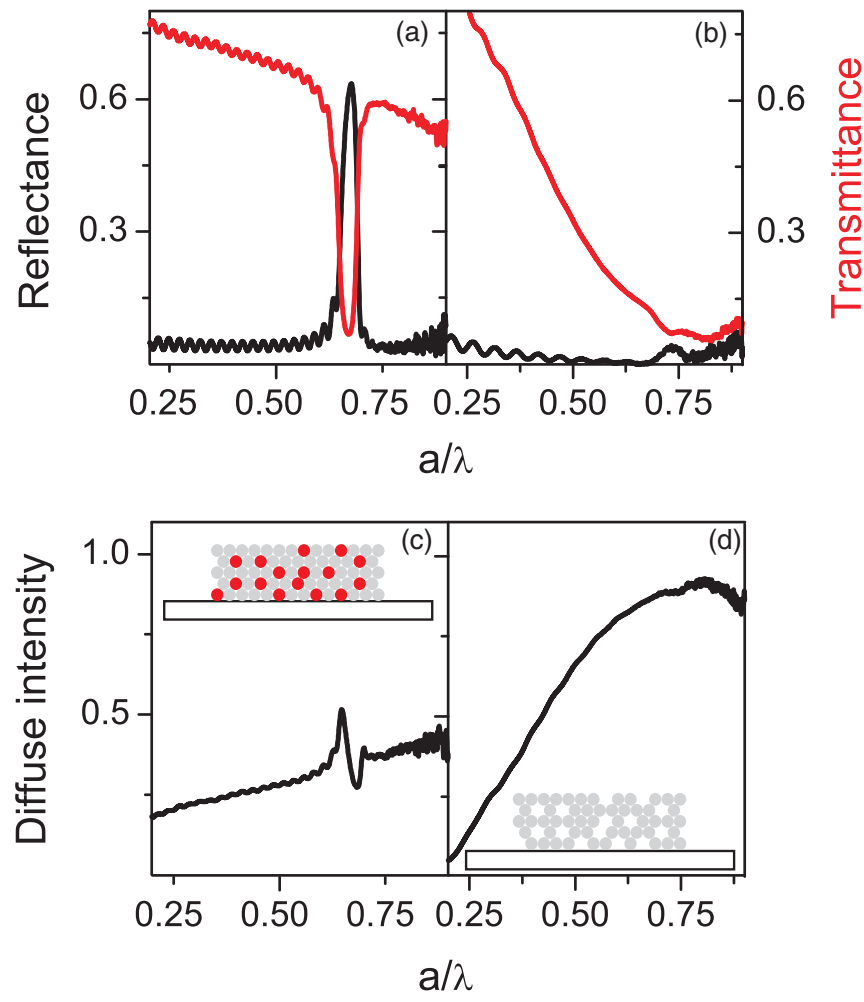

FIG. 3. (Color online) (a) and (b) The reflectance and transmittance spectra measured from samples with $0 \%$ and $40 \%$ vacancy density, respectively. Diffuse light intensity from the corresponding samples (c) before and (d) after PS removal. Notice the presence (disappearance) of the gap before (after) PS spheres removal. The insets in (c) and (d) show a schematical drawing of the corresponding samples.

destructive interference is averaged out giving rise to an effective medium behavior and losses increase monotonically with energy.

\section{STATIC MEASUREMENTS: SCATTERING MEAN FREE PATH}

The measurements of the reflectance and transmittance coefficient, as reported in the previous section, can also be used to determine the scattering mean free path $\ell_{s}$, defined as the length over which a light beam can propagate inside the sample before it is scattered due to randomness [23]. To that end, the reflectance and transmittance are recorded in the way described in Sec. II B, now studied versus the thickness of the sample (see Fig. 4). Specular $R$ and $T$ [similar to those from Figs. 3(a) and 3(b)] are taken in adjacent regions in a set of samples with a vacancy density from $0 \%$ to $40 \%$. Adjacent regions have a different amount of stacked layers and are visible by optical microscope inspection as terraces on the sample surface. The thickness of such films is assessed by measuring the density of Fabry-Perot fringes, which is crucial to provide the exact thickness $L$ of the tested region. The thickness can also be determined in an alternative and independent way by simply counting the terraces on the photonic crystal, since each terrace corresponds to a thickness increase by one layer [see Fig. 4(b)]. The accuracy in the determination of the thickness 

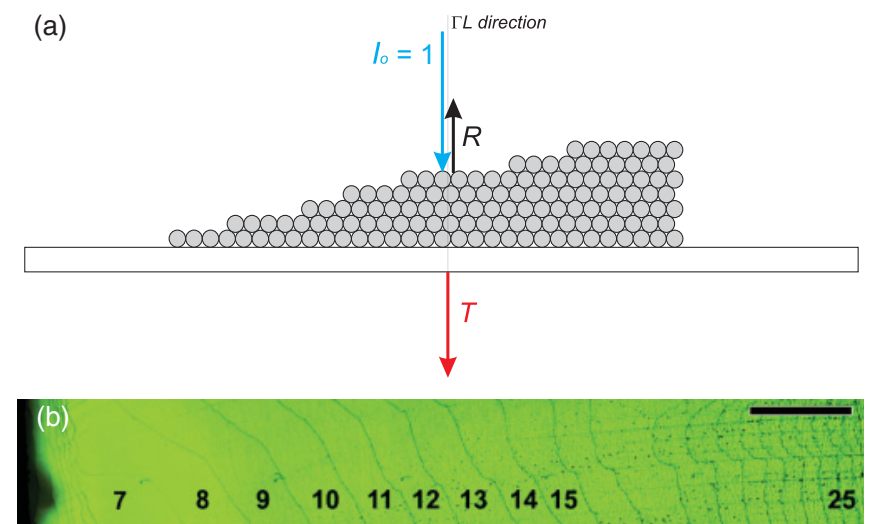

FIG. 4. (Color online) (a) Scheme of the scattering mean free path measurement setup. Specular reflectance and transmittance are measured along the $\Gamma L$ direction (perpendicularly to the sample surface) in adjacent terraces with known thickness. (b) Optical image made combining eight images of the opal surface from a microscope in which the different layers are distinguishable as terraces. The measurements have been performed from 1 to 25 layers, which are clearly distinguishable from each other by eye inspection with the help of the microscope, on adjacent areas, along horizontal lines perpendicular to the crystal growth direction.

is in that case limited by the cumulative effect of the sphere polydispersity $(<2 \%)$.

The analysis of the thickness dependence of reflectance and transmittance spectra has to take into account the fact that light impinging on a photonic crystal can be transmitted, (specularly) reflected, diffracted, absorbed, or (diffusely) scattered. Considering energies below the onset of diffraction $(a / \lambda \sim 1.12)$ [17], diffraction can be disregarded. Absorption is also negligible for the considered frequencies for PS and PMMA spheres. Elastic scattering is then the only loss mechanism and the Lambert-Beer law can be written as

$$
R(L)+T(L)=\exp \left(-\frac{L}{\ell_{s}}\right) .
$$

Figure 5(a) shows the measurement of $\ln (T+R)$ for four different vacancy density at a wavelength $\lambda_{1}=1.6 \mu \mathrm{m}$ for spheres with diameter $d=630 \mathrm{~nm}\left(a / \lambda_{1}=0.56\right)$. In this type of representation, the slope yields directly $\left(-\ell_{s}\right)^{-1}$ according to Eq. (2). Figure 5(c) shows the variation of $\ell_{s}$ with the vacancy density in the passband $\left[\lambda_{1}\right.$, see Fig. 5(b)]. We observe how the optical thickness of the sample $L / \ell_{s}$ (where $L$ is the sample thickness) increases with $\rho_{v}$. Table I shows this evolution and also gives the average number of scattering events light performs before exiting the sample, given by $N=\left(L / \ell_{s}\right)^{2}$. This number is below one $(0.2)$ in the case of the most perfect opal-based photonic crystal which is comparable to other very high quality opals [24] and it becomes as large as 28 in the case of $\rho_{v}=40 \%$. This last value is comparable to opals grown by centrifugation [25] which show an average number of scattering events of $\sim 15$. The very high quality of the crystals is of paramount importance to access the different light transport regimes of our samples: near-single scattering in the case of nondoped crystals up to diffusion in the highest doping case of $\rho_{v}=40 \%$.
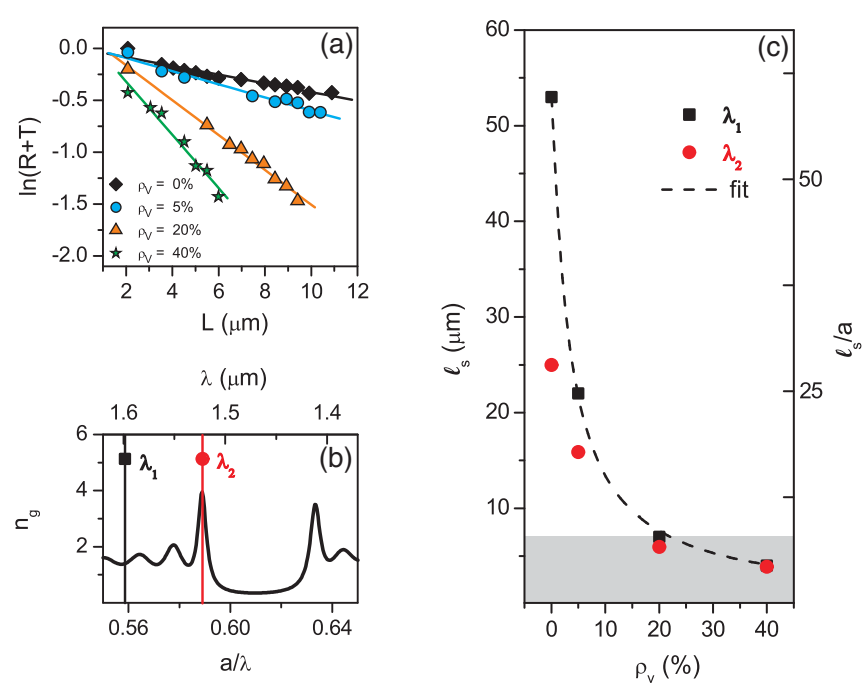

FIG. 5. (Color online) Measurement of the scattering mean free path in photonic crystals. (a) Plot of $\ln (R+T)$ as a function of the sample thickness $L$ at $\lambda_{1}=1.67 \mu \mathrm{m}$ for different vacancy density (from $0 \%$ to $40 \%$ ). These photonic crystals are made of PMMA spheres of $630 \mathrm{~nm}$ diameter and refractive index $n=1.4$. The inverse of the slope yields directly the scattering mean free path. (b) Calculated group index $n_{g}$ for an ideal photonic crystal of 40 layers thickness along the $\Gamma L$ direction for the case of $\rho_{v}=0 \%$. The scattering mean free path $\ell_{s}$ is measured in the passband $\left(a / \lambda_{1}=0.56\right.$, black squares $)$ and at the band edge $\left(a / \lambda_{2}=0.59\right.$, red circles) as a function of the vacancy density $\rho_{v}$. In (c), the scattering mean free path is plotted vs vacancy density and compared to the Bragg length ( $L_{B}$, shaded area) in the case of $\rho_{v}=0 \%$. The variation of $\ell_{s}$ is smaller at the band edge than in the passband. The black dashed curve represents the fit of $\ell_{s}$ vs $\rho_{v}$ at the passband.

From Figs. 5(b) and 5(c) it is clear that the dependence of the scattering mean free path is very different at a wavelength at the band edge ( $\lambda_{2}$ in Fig. 5) than it is at wavelengths far away from the stopband ( $\lambda_{1}$ in Fig. 5). At the band edge the density of states is high and the group velocity low, which increases the amount of scattering and hence reduces the scattering mean free path $[23,26]$. This explains the large difference between $\ell_{s}$ at the two wavelengths, for $\rho_{v}=0 \%$. At increasing $\rho_{v}$, the effect of the photonic crystal diminishes and at $\rho_{v}=40 \%$ there is no difference in the value of the scattering mean free path for the two wavelengths. At wavelengths far away from the stopband, we do not expect strong effects from the photonic crystal and the inverse of the scattering mean free path should simply depend linearly on the density of scattering elements. If we distinguish between the scattering that is intrinsically present in the photonic crystal (expressed in terms of a density $\rho_{0}$ and cross section $\sigma_{0}$ that represents the average of all

TABLE I. Optical thickness.

\begin{tabular}{lcccc}
\hline \hline$\rho_{v}(\%)$ & $L_{B}(\mu \mathrm{m})$ & $\ell_{s}(\mu \mathrm{m})$ & $L / \ell_{s}$ & $N$ \\
\hline 0 & $8 \pm 1$ & $53 \pm 4$ & 0.4 & 0.2 \\
5 & & $22 \pm 2$ & 1 & 1 \\
20 & $7.0 \pm 0.7$ & 3 & 9 \\
40 & & $4.0 \pm 0.4$ & 5.3 & 28 \\
\hline \hline
\end{tabular}


intrinsic scattering, for example, due to polydispersity of the spheres, cracks, staking faults, and so on), and the scattering introduced by the vacancies (with vacancy density $\rho_{v}$ and vacancy scattering cross section $\sigma_{v}$ ), we can write the inverse of the scattering mean free path as

$$
\ell_{s}^{-1}=\rho_{0} \sigma_{0}+\rho_{v} \sigma_{v}
$$

assuming independent scattering from the vacancies. Equation (3) allows us to fit $\ell_{s}\left(\rho_{v}\right)$ and extract the value $\sigma_{v}=$ $(0.057 \pm 0.002) \mu \mathrm{m}^{2}$. Note that at both wavelengths $\lambda_{1}$ and $\lambda_{2}$ the mean free path becomes smaller than the Bragg length for $\rho_{v}>20 \%$ [shaded area in Fig. 5(c)], which means that above those vacancy densities the Bloch approximation fails to give an accurate description of the propagation process.

\section{DYNAMIC MEASUREMENTS: DIFFUSION CONSTANT}

The static measurements presented in Sec. III reveal how sensitive $\ell_{S}$ is to the vacancy density. In order to get information on the behavior of the diffusion constant, one has to resort to time-resolved measurements. In this section we will use a nonlinear optical gating technique to analyze the time-resolved response of transmitted diffuse light through photonic crystals with vacancies [27]. This will allow us to measure the diffusion constant as a function of wavelength and disorder.

Most regular disordered systems are isotropic, meaning that the diffusion constant and mean free path are angular independent. In (partially disordered) photonic crystals it is, on the contrary, crucial to take into account directionality. The photonic band edge of a stopband, for instance, occurs for a wavelength which will change when varying angle, hence its effect on the diffuse transport of light can also be anisotropic. The technique that we use in this section to measure the time evolution of the transmitted diffuse light is sensitive to the component of the diffusion constant in the direction perpendicular to the slab, which is also the direction in which $\ell_{s}$ has been determined in the static measurements described in the previous section.

Figure 6 shows time-resolved transmission through thin film opals, thickness $21 \mu \mathrm{m}$ ( $\sim 40$ layers), grown with PMMA spheres (diameter $d=630 \mathrm{~nm}$ ) for $\rho_{v}=0 \%$. The measurements have been performed at $a / \lambda_{1}=0.56$ (black dotted curve) and $a / \lambda_{2}=0.59$ (red dotted curve). The reference pulse is plotted with a blue solid curve in both figures to compare it with the diffuse decay. The value of the diffusion constant is obtained by fitting the experimental time profile of $T(t)$ with the dynamical solution to the diffusion equation [28]. At the band edge we obtain $\mathcal{D}\left(\lambda_{2}\right)=220 \mathrm{~m}^{2} / \mathrm{s}$ while in the passband, at $\lambda_{1}$, the transmitted pulsed through the sample is of the order of the probe pulse (150 fs, blue solid curve). This is due to the fact that $\mathcal{D}\left(\lambda_{1}\right)$ is larger than the maximum diffusion constant we can measure with our setup $\left(\mathcal{D}_{\max } \sim 700 \mathrm{~m}^{2} / \mathrm{s}\right)$.

The optical thickness of the sample $L / \ell_{s}$ is wavelength dependent at $\rho_{v}=0 \%$, as shown in the previous section. In the passband, $\ell_{s}\left(\lambda_{1}\right)$ acquires a very large value compared to $L$ and $\mathcal{D}\left(\lambda_{1}\right)$ becomes very large as well. At this wavelength, scattering becomes dominantly single and low-order scattering so that it is actually not meaningful anymore to define a diffusion constant in the first place. The observed strong wavelength dependence diminishes at increasing disorder.

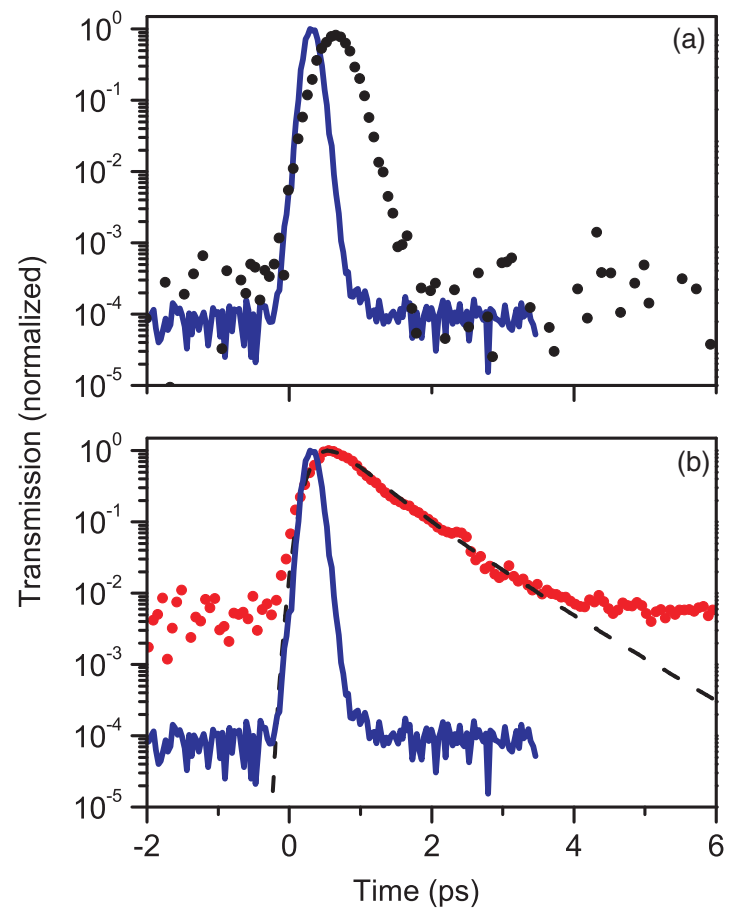

FIG. 6. (Color online) Plot of the time-resolved diffuse transmission through photonic crystals composed by PMMA spheres with $n=1.42$ and a diameter $d=630 \mathrm{~nm}$ with $\rho_{v}=0 \%$ at the passband, $a / \lambda_{1}=0.56$ black dotted curve (a), and at the band edge, $a / \lambda_{2}=0.59$ (b) red dotted curve. The blue solid curve is the time-resolved transmission of the pulse reference. The black dashed line represents the fit of the experimental measurement with the diffusion equation.

This is shown in the inset of Fig 7 and is expected, as the photonic crystal correlations disappear. This behavior is clearly observed in the inset of Fig. 7 where $\mathcal{D}$ is plotted at $\rho_{v}=40 \%$.

The complete measurement of $\mathcal{D}\left(\lambda_{2}\right)$ as a function of $\rho_{v}$ is plotted in Fig. 7. It shows a fivefold decrease of the value of $\mathcal{D}$ as a consequence of disorder from $\mathcal{D}(0 \%)=220 \mathrm{~m}^{2} / \mathrm{s}$ to $\mathcal{D}(40 \%)=43 \mathrm{~m}^{2} / \mathrm{s}$. The diffusion constant already reaches its minimal value at $20 \%$ vacancy density and increasing the vacancy density beyond that point seems not to reduce the diffusion constant further. The total decrease of $\mathcal{D}$ at $\lambda_{2}$ is comparable to the decrease of $\ell_{s}$ at the same wavelength.

The diffusion constant in regular isotropic disordered systems is given by

$$
\mathcal{D}=\frac{1}{3} \ell_{t} v_{e},
$$

where $v_{e}$ is the energy velocity [29] and $\ell_{t}$ is the transport mean free path. This relation remains valid in isotropic systems for each Cartesian coordinate if we take the appropriate values for $\mathcal{D}, \ell_{t}$, and $v_{e}$ in those directions. As mentioned above, our experimental technique is sensitive to the value of the diffusion constant in the direction perpendicular to the slab, which is also the direction in which we have determined the other optical properties of our samples.

If we now assume, to first order, $\ell_{t} \approx \ell_{s}$ (which is reasonable for our system) we can use the values of $\ell_{s}(40 \%)$ and $\mathcal{D}(40 \%)$ to estimate $v_{e}$, which yields $v_{e}(40 \%) \sim 0.25 c$ (where $c$ is the speed of light in vacuum). This value is small 


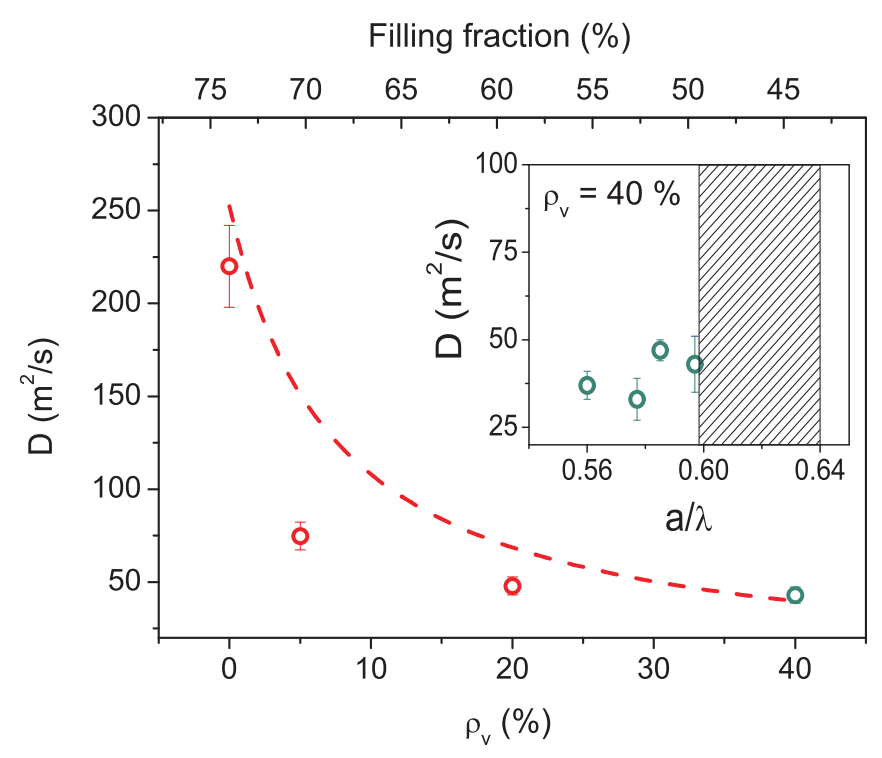

FIG. 7. (Color online) Diffusion constant $\mathcal{D}$ as a function of the vacancy density $\rho_{v}$ measured at the band edge $a / \lambda_{2}=0.59$. The red dashed curve represents the expected value of $\mathcal{D}$ when assuming a constant energy velocity and using the vacancy dependence of the mean free path $\ell_{s}\left(\rho_{v}\right)$ as found before. One can see that the values at high and low vacancy density are correctly predicted this way, but the diffusion constant at intermediate vacancy densities is overestimated. The error bars are normally assigned varying the initial parameters of the fit, such as the sample thickness, in agreement with the experimental uncertainty, and recording correspondingly the interval of possible values for $\mathcal{D}$. They typically present a relative error of $5 \pm 40 \%$. In the inset of the figure the measured $\mathcal{D}$ is plotted as a function of energy for $\rho_{v}=40 \%$ (the position of the pseudogap is represented by the dashed area).

compared to the phase velocity in an equivalent homogeneous system with the same average refractive index as our materials, which lies between 1.3 and 1.4, and also much smaller then the transport velocity that one would expect for the equivalent fully disordered system.

In Fig. 7 we have also plotted the vacancy density dependence of the diffusion constant if we assume the transport velocity to remain constant, using only the vacancy dependence of the mean free path $\ell_{s}\left(\rho_{v}\right)$ as found before. We see that the overall variation of the diffusion constant of a factor of 5 can be entirely explained in that case by the vacancy dependence of $\ell_{s}\left(\rho_{v}\right)$. However, at intermediate vacancy values the such obtained curve overestimates $\mathcal{D}$. Currently there is no theory available that can describe accurately the behavior of the transport velocity in partially disordered photonic crystals, and this would be an interesting topic to look into in the future.

\section{TOTAL WHITE LIGHT TRANSMISSION: FROM CRYSTALS TO GLASSES}

In the previous sections we have shown via static and dynamic measurements how a high amount of vacancies gives rise to strong multiple scattering in photonic crystals. In this section we want to compare these highly doped photonic crystals with photonic glasses. To this purpose, we measured total light transmission from 400 to $900 \mathrm{~nm}$ wavelength

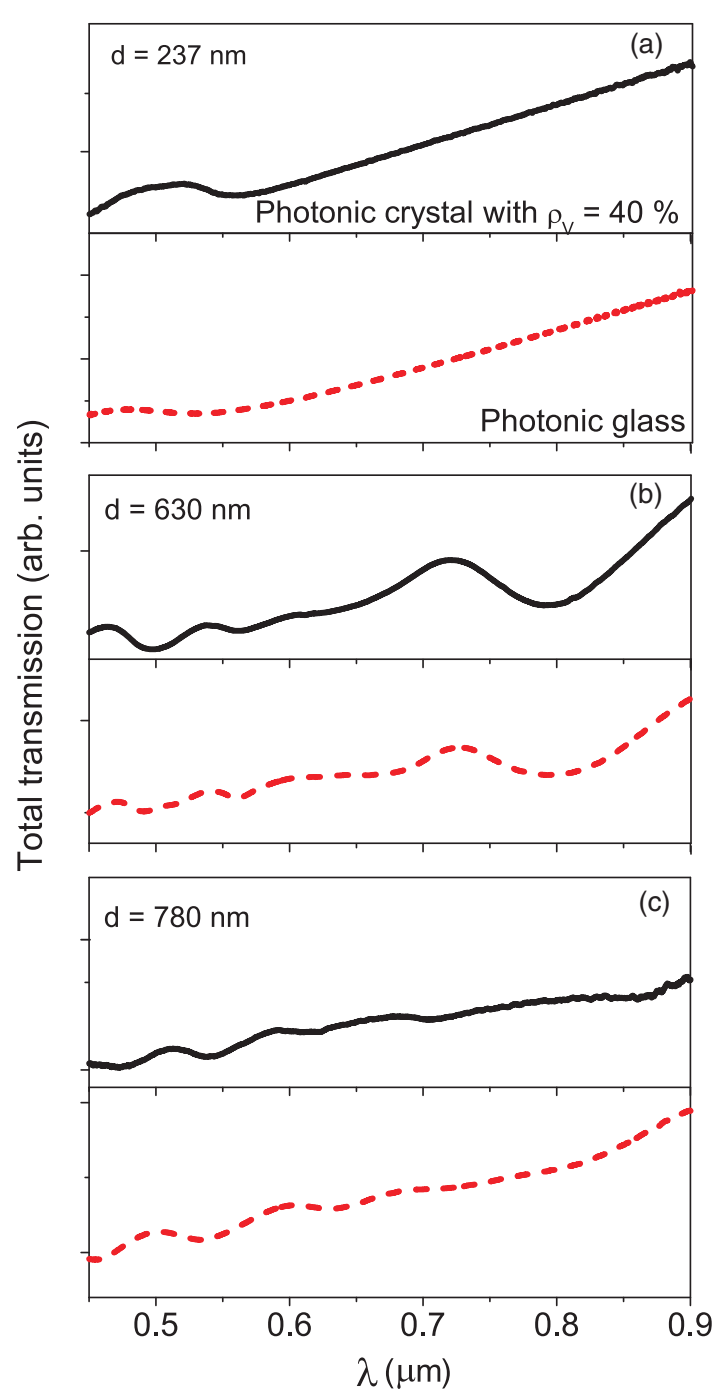

FIG. 8. (Color online) Total light transmission through photonic crystals with $40 \%$ vacancies (black solid curves) compared to photonic glasses made with the same spheres (red dashed curves) with diameters (a) $d=237 \mathrm{~nm}$, (b) $d=630 \mathrm{~nm}$, and (c) $d=780 \mathrm{~nm}$.

through photonic crystals with $\rho_{v}=40 \%$ with an integrating sphere (the setup is shown elsewhere [22]) on large areas of $\sim 1 \mathrm{~mm}^{2}$.

Figure 8 compares total light transmission through photonic crystals with $\rho_{v}=40 \%$ vacancies and through photonic glasses made with the same PMMA spheres for three different diameters: (a) $d=237 \mathrm{~nm}$, (b) $d=630 \mathrm{~nm}$ and (c) $d=$ $780 \mathrm{~nm}$. The three figures show that the total transmission presents a very similar behavior in both cases, including the occurrence of very similar resonances. The transport mean free path measured in photonic glasses, $\ell_{t} \simeq 3 \mu \mathrm{m}$ [8] is comparable to the one measured in natural-sedimented photonic crystals [30] and to the value of $\ell_{s}$ obtained previously for our photonic crystals with $\rho_{v}=40 \%$ vacancies $\ell_{s} \simeq 4 \mu \mathrm{m}$. The occurrence of the same resonances in the total transmission for photonic glasses and photonic crystals with vacancies is noteworthy. In photonic glasses these resonances originate from the (Mie) resonances in the single scattering coefficient of the monodisperse constituent spheres. In the doped 
photonic crystals, the scattering originates from vacancies and apparently, at high vacancy density, the Mie resonances of the spheres that constitute the photonic crystal emerge as dominant when the spatial correlations of the photonic crystal are strongly attenuated. The remarkable similarity of the curves shown in Fig. 8 is a clear indication of the convergence of a photonic crystal into a photonic glass with disorder.

\section{CONCLUSIONS}

In this paper we report on the realization of photonic crystals with a controlled amount of, well-defined, disorder, the number density of vacancies. We characterize the optical properties of such materials in various ways and determine the degree of scattering at various wavelengths and doping concentrations. In particular, our measurements show that it is possible to control and fine tune the amount of multiple scattering in a photonic crystal by adding vacancies and hence without altering the crystal structure. In the highly vacancy doped photonic glass we observe a resonant behavior, analogous to that observed due to Mie scattering in photonic glasses. Our materials might therefore be useful to explore Fano-like interactions [31] between the extended Bloch mode of the photonic crystal and spatially confined Mie modes.

The possibility of controlling light scattering and diffusion in photonic crystals has important implications to test and understand better the quality of photonic crystal-based devices. The use of high refractive index materials like Si [32] may amplify the effect presented here becoming proper candidates to observe and control Anderson localization of light in 3D [33], as already observed in slightly disordered 1D photonic crystal wave guides [34,35] and for the spectral control of lasing emission from ordered or disordered active media.

\section{ACKNOWLEDGMENTS}

We thank J. F. Galisteo-Lopez for the data of the group index. The work was supported by the EU through Network of Excellence IST-2-511616-NOE (PHOREMOST), and partially supported by EU FP7 NoE Nanophotonics4Energy Grant No. 248855; the Spanish MICINN CSD2007-0046 (Nanolight.es), MAT2009-07841 (GLUSFA) and Comunidad de Madrid S2009/MAT-1756 (PHAMA) projects. RS acknowledges support by RyC.
[1] E. Yablonovitch, Phys. Rev. Lett. 58, 2059 (1987).

[2] S. John, Phys. Rev. Lett. 58, 2486 (1987).

[3] A. F. Koenderink and W. L. Vos, Phys. Rev. Lett. 91, 213902 (2003).

[4] J. F. Galisteo-López, M. Ibisate, R. Sapienza, L. S. Froufe-Pérez, A. Blanco, C. López, Adv. Mater. 23, 30 (2011).

[5] P. V. Braun, S. A. Pruzinsky, and F. Garcia-Santamaria, Adv. Mater. 18, 2665 (2006).

[6] Y. Akahane, T. Asano, B. Song, and S. Noda, Nature (London) 425, 944 (2003).

[7] P. D. Garcia, R. Sapienza, and C. Lopez, Adv. Mater. 22, 12 (2010).

[8] R. Sapienza, P. D. Garcia, J. Bertolotti, M. D. Martin, A. Blanco, L. Vina, C. Lopez, and D. S. Wiersma, Phys. Rev. Lett. 99, 233902 (2007).

[9] P. W. Anderson, Phys. Rev. 109, 1492 (1958).

[10] C. Toninelli, E. Vekris, G. A. Ozin, S. John, and D. S. Wiersma, Phys. Rev. Lett. 101, 123901 (2008).

[11] T. Schwartz, G. Bartal, S. Fishman, and S. Mordechai, Nature (London) 446, 52 (2007).

[12] K. P. Velikov, C. G. Christova, R. P. A. Dullens, and A. van Blaaderen, Science 296, 106 (2002).

[13] V. Kitaev and G. Ozin, Adv. Mater. 15, 75 (2003).

[14] D. Wang and H. Moehwald, Adv. Mater. 16, 244 (2004).

[15] P. Jiang, J. F. Bertone, K. S. Hwang, and V. L. Colvin, Chem. Mater. 11, 2132 (1999).

[16] M. Born and E. Wolf, Principles of Optics (Pergamon, New York, 1986).

[17] J. F. Galisteo-Lopez, E. Palacios-Lidon, E. Castillo-Martinez, and C. Lopez, Phys. Rev. B 68, 115109 (2003).

[18] V. N. Astratov, A. M. Adawi, S. Fricker, M. S. Skolnick, D. M. Whittaker, and P. N. Pusey, Phys. Rev. B 66, 165215 (2002).

[19] This consideration is valid for our samples, since PS and PMMA absorbance is negligible for the considered frequencies [20,21], confirmed also by measurements of the absorption length $\left(\ell_{\mathrm{a}} \sim 10 \mathrm{~m}\right)$ in the visible range for a photonic glass composed by the same polymer spheres [22]. Higher order scattering is also negligible compared to the scattering losses.

[20] T. Inagaki, E. T. Arakawa, R. N. Hamm, and M. W. Williams, Phys. Rev. B 15, 3243 (1977).

[21] M. A. Khashan and A. Y. Nassif, Opt. Commun. 188, 129 (2001).

[22] P. D. Garcia, R. Sapienza, J. Bertolotti, M. D. Martin, A. Blanco, A. Altube, L. Vina, D. S. Wiersma, and C. Lopez, Phys. Rev. A 78, 023823 (2008).

[23] P. D. Garcia, R. Sapienza, L. S. Froufe-Perez, and C. Lopez, Phys. Rev. B 79, 241109(R) (2009).

[24] J. J. Baumberg, O. L. Pursiainen, and P. Spahn, Phys. Rev. B 80, 201103(R) (2009).

[25] M. D. Birowosuto, Otto L. Muskens, A. Femius Koenderink, and Willem L. Vos, Phys. Rev. B 83, 155101 (2011).

[26] L. S. Froufe-Perez, R. Sapienza, P. D. Garcia, and C. Lopez, (in preparation).

[27] R. Sapienza, P. Costantino, D. S. Wiersma, M. Ghulinyan, C. J. Oton, and L. Pavesi, Phys. Rev. Lett. 91, 263902 (2003).

[28] M. S. Patterson, B. Chance, and B. C. Wilson, Appl. Opt. 28, 2331 (1989).

[29] M. P. van Albada, B. A. van Tiggelen, A. Lagendijk, and A. Tip, Phys. Rev. Lett. 66, 3132 (1991).

[30] A. F. Koenderink, M. Megens, G. van Soest, W. L. Vos, and A. Lagendijk, Phys. Lett. A 268, 104 (2000).

[31] M. V. Rybin, A. B. Khanikaev, M. Inoue, K. B. Samusev, M. J. Steel, G. Yushin, and M. F. Limonov, Phys. Rev. Lett. 103, 023901 (2009)

[32] M. Ibisate, D. Golamayo, and C. Lopez, Adv. Mater. 21, 2899 (2009).

[33] S. John and M. J. Stephen, Phys. Rev. B 28, 6358 (1983).

[34] L. Sapienza, H. Thyrrestrup, S. Stobbe, P. D. Garcia, S. Smolka, and P. Lodahl, Science 327, 1352 (2010).

[35] P. D. Garcia, S. Smolka, S. Stobbe, and P. Lodahl, Phys. Rev. B 82, 165103 (2010). 